\title{
KIAA1199 induces advanced biological behavior and development of ovarian cancer through activation of the IL-6/STAT3 pathway
}

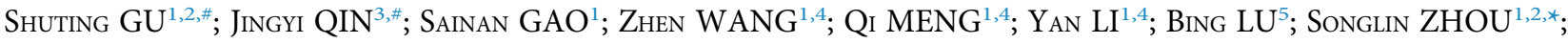 \\ YUNZHAO XU $\mathrm{XU}^{1,6, *}$ \\ ${ }^{1}$ Department of Obstetrics and Gynecology, Affiliated Hospital of Nantong University, Nantong, 226001, China \\ 2 Key Laboratory of Neuroregeneration of Jiangsu and Ministry of Education, Jiangsu Clinical Medicine Center of Tissue Engineering and Nerve Injury Repair, \\ Co-Innovation Center of Neuroregeneration, Nantong University, Nantong, 226001, China \\ ${ }^{3}$ Key Laboratory for Nano-Bio Interface Research, Nano-Bio-Chem Centre, Suzhou Institute of Nano-Techand Nano-Bionics, Chinese Academy of Sciences, \\ Suzhou, 215123, China \\ ${ }^{4}$ Department of Obstetrics and Gynecology, Dalian Medical University, Dalian, 116044, China \\ ${ }^{5}$ Department of Clinical Biobank, Affiliated Hospital of Nantong University, Nantong, 226001, China \\ ${ }^{6}$ Department of Obstetrics and Gynecology, School of Medicine, Nantong University, Nantong, 226001, China
}

Key words: Epithelial Ovarian Cancer (EOC), KIAA1199, IL-6/STAT3 pathway, Prognosis

\begin{abstract}
Recently, abnormal expression of KIAA1199 has been detected in Epithelial Ovarian Cancer (EOC). However, the underlined anti-ovarian cancer mechanism of KIAA1199 remains to be enlightened. In our study, we performed to elucidate the effects of KIAA1199 on the advanced biological behavior of EOC cells through activation of the IL-6/STAT3 pathway. Confirmed by immunohistochemistry, KIAA1199 was highly expressed in ovarian borderline and malignant epithelial tumors. A retrospective analysis found that EOC patients with low expression of KIAA1199 had a significantly higher 5-year survival rate than those with high expression. Mechanistically, IL-6 was used to stimulate EOC cells, and the expression of KIAA1199, STAT3 and p-STAT3 increased after IL-6 stimulation. These results could show that KIAA1199 is transcriptionally activated by IL6/STAT3 pathway, thereby accelerating the deterioration of EOC. KIAA1199 could also be used as a poor prognosis factor and potential target in treatment.
\end{abstract}

\section{Introduction}

As a malignant reproductive system tumor that poses a serious risk to women's health, ovarian cancer's fatality rate ranks the first among all the gynecological malignancies (Lheureux et al., 2019). Ovarian cancer is mainly epithelial cancer with insidious onset (Kroeger and Drapkin, 2017). Most patients have reached an advanced stage when they visit outpatient, with the tumor developing rapidly, and showing spreads and distant metastases, mainly manifested by the dissemination of intra-abdominal lesions, and the prognosis being poor (Lheureux et al., 2019). There are many factors that may affect the malignancy and prognosis of ovarian cancer, such as changes in ECM, tumor stem cells, and tumor-promoting microenvironment, etc.,

\footnotetext{
*Address correspondence to: Yunzhao Xu, xuyz@ntu.edu.cn; Songlin Zhou, songlin.zhou@ntu.edu.cn

${ }^{\#}$ These authors contributed equally to this work

Received: 19 February 2021; Accepted: 24 May 2021
}

(Da Silva et al., 2020; Rooth, 2013; Yang et al., 2020). Currently, first-line treatments for EOC involve debulking surgery followed by chemotherapy and target therapy (Guan and Lu, 2018). Even though this initial therapy shows a better curative effect on more than $80 \%$ of the patients, chemotherapy resistance will appear when most patients relapse (Lee et al., 2019). A better understanding of tumorigenic mechanism is of great significance for the development of therapeutic targets (Arend et al., 2021; Nimmagadda and Penet, 2019) for EOC patients.

KIAA1199, also known as cell migration-inducing hyaluronan binding protein (CEMIP), is a primary depolymerase of hyaluronic acid (HA) which is an essential constituent of extra-cellular matrix (ECM) (Yoshida and Okada, 2019). KIAA1199 is an important depolymerase of HA with a molecular weight of about $150 \mathrm{kDa}$. There is a secretion signal peptide at its $\mathrm{N}$-terminus which can promote its effective secretion to the outside of the cell (Yoshida et al., 2014). KIAA1199 is a multifunctional protein that is mainly located in the endoplasmic reticulum 
in cells and regulates the release of calcium ions in the endoplasmic reticulum, thereby promoting the activation of calcium-related signal proteins (Zhang et al., 2014). Although it was initially identified as a congenital ear congenital disease-causing gene (Abe et al., 2003), it has been shown through many studies that the KIAA1199 maintains a close association with the progression of tumors and is involved in a variety of physiological and pathological processes (Zhang et al., 2014). During the pathogenesis of Crohn, the expression of KIAA1199 in ECM is significantly increased, and numerous low molecular weight HA fragments can be detected in diseased tissues (Soroosh et al., 2016). These fragments are believed to aggravate the occurrence of chronic inflammation and the secretion of IL-6, then ultimately aggravate the condition. However, interference with KIAA1199 can inhibit the above process.

Previous studies have indicated that over-expressed KIAA1199 could be detected under a variety of pathological conditions, such as cell migration, chronic inflammation, and hypoxic microenvironment, which suggests that KIAA1199 has a complex regulatory mechanism. Especially, related studies have suggested that KIAA1199 bears a major responsibility in a variety of signaling pathways, such as Wnt and FGFR (Li et al., 2017). IL-6/STAT3 signaling pathway is closely related to tumorigenesis and also may play the part of a promising target to strengthen tumor immunotherapy's efficacy (Kitamura et al., 2017). IL-6 is a multi-potency cytokine, which shapes IL-6/sIL-6R complex by binding to soluble IL-6 receptor, and then activates gpl30 on the cell membrane surface to activate and maintain the phosphorylation of STAT3 (Garbers et al., 2015; Mihara et al., 2012). STAT3 is an important member of the STATs family and plays an important role in immune cells and tumor cells. Activated STAT3 modulates the expression of vital key genes, thereby promoting cell proliferation, migration and invasion, anti-apoptosis, etc., and then exhibiting carcinogenic effects (Johnson et al., 2018). Based on the above, we aimed to illustrate whether KIAA1199 could promote tumorigenesis of EOC by the activation of IL-6/STAT3 pathway within the present study.

\section{Materials and Methods}

Clinical specimens and patient clinical information

Borderline ovarian epithelial tumors, epithelial ovarian cancer, and normal ovarian tissue specimens were obtained from patients undergoing gynecological surgery in the Affiliated Hospital of Nantong University who had given their consent before. The specimens of ovarian tumors were independently confirmed by two chief physicians of the pathology department in our hospital. There were 10 cases of normal ovarian tissues and 36 cases of borderline ovarian epithelial tumor samples, and 126 cases of ECO samples. The 126 patients with ovarian cancer selected in the retrospective analysis were all from our hospital who underwent surgery. At the same time, the experiment had received the approval from the ethics committee of the Affiliated Hospital of Nantong University (No. 2018-L005, DATE: 05/03/2018). Follow-up information on the study in which 7 patients were lost to follow-up was updated through Dec. 31, 2018, by reviewing medical records and data from the Chinese Public Security Bureau.

\section{Immunohistochemical staining and analysis}

We performed immunohistochemistry (IHC) to detect the KIAA1199 protein expression by using Tissue Microarrayer System Quick Ray (UT06, UNITMA, Korea) in 126 cases of EOC paraffin sections. The sections were deparaffinized, ethanol gradient rehydrated, and antigen retrieval was performed. KIAA1199 immunostaining on TMAs (Tissue microarrays) was observed by anti-KIAA1199 antibody (1:100, Novus, NBP2-50336, USA) as the primary antibody at $4^{\circ} \mathrm{C}$ overnight, and then incubated with the anti-mouse secondary antibody (1:100, Santa Cruz, SC-2039, CA, USA) at $37.0^{\circ} \mathrm{C}$ for $30 \mathrm{~min}$. KIAA 1199 semiquantitative expression of each slide was evaluated and scored by two independent pathologists who were unaware of the clinical characteristics of the cases, taking the staining intensity into account, which was scored as 0 (no staining), 1+ (weak staining), 2+ (medium staining), or $3+$ (strong staining).

Cell lines and IL-6 stimulation and Cryptotanshinone disposal Human EOC cell lines HO8910, A2780 and CAOV-3 cells were purchased from National Collection of Authenticated Cell Culture. All cells were cultured in RPMI-1640, within which $10 \%$ fetal bovine serum (FBS) and $1 \%$ penicillin/ streptomycin antibiotic mixture were added. Another human EOC cell line OVCAR-3 was cultured in DMEM supplemented with $10 \%$ FBS and $1 \%$ antibiotic mixture. All of them were cultured under a humid environment of $5 \%$ $\mathrm{CO}_{2}$ at $37^{\circ} \mathrm{C}$.

Cells HO8910 adjusted to the concentration at $5 \times 10^{5}$ cells $/ \mathrm{mL}$ were seeded to 6 -well plates at $2 \mathrm{~mL} /$ well. Twentyfour hours later, the medium was withdrawn and substituted in the presence of IL-6 (200 ng/mL, H4218, ACROBiosystems) or Cryptotanshinone (STAT3 pathway inhibitor) $(10 \mu \mathrm{M}, \mathrm{M} 3982$, Abmole) dissolved in DMSO or DMSO for specific time points. All our experiments were carried out three times independently.

\section{Western blotting}

Radioimmunoprecipitation (RIPA) lysis buffer containing PMSF was used to extract the cell sample protein, of which the experiment procedure was performed as described previously (Sheng et al., 2018). The rabbit anti-human KIAA1199 (ab98947, Abcam, 1:500), anti-CEMIP (cell migration-inducing protein, 1:1000, ABclonal, Woburn, MA, USA), p-STAT3 (Cell signaling Technology, \#9145, 1:2000), STAT3 (Santa Cruz, SC-8019, 1:1000) polyclonal antibody were used as primary antibodies. the PVDF membranes were incubated with the HRP-conjugated goat anti-rabbit IgG as secondary antibody (Proteintech, SA00001-1, SA00001-2, 1:5000). Results were analyzed using Image J 2X software (NIH, Bethesda, MD, USA).

\section{RT-PCR}

Total RNA was extracted with Trizol extraction kit (Invitrogen), and cDNA was synthesized utilizing the SYBRGreen PCR kit (Thermo F-415XL) according to the 
operation instructions. KIAA1199 primer: forward 5'-AGTGATGGGGGAGATGGAGG-3'; reverse 5'-GCCAGGTGGAAGTGAATCGG-3'. GAPDH primer: forward 5'-AGAAGGCTGGGGCTCATTTG-3'; reverse 5'-AGGGGCCATCCACAGTCTTC-3'.

Real-time quantitative PCR (RT-qPCR) was performed with the FastStart Universal SYBR Green Master (ROX) (Roche, Basel, Switzerland) and the Light Cycler Nano realtime fluorescence quantification PCR system (Roche, USA).

\section{KIAA1199 silencing and Invasion and migration assays}

Four different shRNA sequences for KIAA1199 silencing and a negative control were designed as below: shRNA-1, sense 5'GCAATCGTCCCATTGATATAC-3', shRNA-2, sense 5'-GCTGCAGGATCTGAGGAAACT-3', shRNA-3, sense 5'GGTTATGACCCACCCACATAC-3', shRNA-4, sense 5'-GGGATAAGACATCTGTGTTCC-3', shRNA-NC, sense 5'-TTCTCCGAACGTGTCACGT-3'. Following instructions of the manufacturers, cells were transfected with Lipofectamine ${ }^{\mathrm{TM}} 3000$ ( $3 \mu \mathrm{L} /$ well, Invitrogen). Transwell Boyden chambers (Corning, NY, USA) were applied to evaluate cell invasion and migration ability of cells. The details of experiment procedure were described previously (Sheng et al., 2018).

\section{Chromatin immunoprecipitation}

Magna RIP RNA Binding Protein Immunoprecipitation Kit (P2078, Beyotime) was used to carry out the RIP assay in the light of the instructions of manufacturers. Entire cell lysate was utilized for immunoprecipitation with STAT3 antibody (SC-8019, Santa Cruz). Rabbit IgG antibody (SA00001-2, Proteintech) was used as the control.

Supernatants $(0.2 \mathrm{~mL})$ underwent immunoprecipitation overnight with antibodies against STAT3 $(5 \mu \mathrm{g})$ at $4^{\circ} \mathrm{C}$ overnight. Chromatin-antibody complexes were separated by Protein A/G PLUS agarose. Before phenol/chloroformpurification, reverse the crosslinks for the enriched and the input DNA and clean the DNA with RNase A and proteinase K. For quantification of the enrichment of KIAA1199 at promoter regions, the ABI 7500 Real-Time PCR System (Applied Biosystems) was used to carry out qRT-PCR.

\section{Statistical analysis}

SPSS20 and STATA12.0 statistical software were used for data analysis. Data were showed as mean \pm standard deviation (SD). T-test was performed to identify differences between variables and control groups. $\mathrm{X}^{2}$ tests were performed to access different expression of KIAA1199 between EOC and non-malignant tissues and whether KIAA1199 expression was correlated with clinicopathologic factors. Kaplan-Meier method was applied to determine survival. Significance was determined by log-rank test. A statistically significance was regarded to indicate when $P<0.05$.

\section{Results}

KIAA1199 expression in EOC tissues and non-malignant tissues KIAA1199 was detected by Immunohistochemical analysis in tissues obtained from 126 cases of EOC patients and cases of and 36 borderline ovarian epithelial tumor and 10 cases of normal ovarian tissues, which demonstrated that KIAA1199 protein expression was considerably higher in EOC tissues than non-malignant ones, which mainly located in the cytoplasm and cell membrane $(P<0.05)$ (Fig. 1$)$. The data showed statistical significance using $\mathrm{X}^{2}$ test analysis $\left(\mathrm{X}^{2}=28.270, P<0.001\right)$ (Table 1$)$.

Relationship between KIAA1199 and clinicopathologic factors of EOC patients

When detected the relationship between KIAA1199 and clinicopathologic factors of EOC patients, we assigned patients into two groups: one was KIAA1199 protein high expression group $(++,+++)$, and the other was KIAA1199 protein negative or low expression group $(-,+)$ according to the immunohistochemical staining of KIAA1199 (Table 2). It has been shown from the results that the higher KIAA1199 expression was related to advanced FIGO stage $(2=67.587$; $P=0.000)$, the presence of extrapelvic metastasis $(2=27.108$; $P=0.000)$ and liver metastasis $(2=9.229 ; P=0.026)$.

Survival analysis between KIAA1199 expression and EOC patients

Kaplan-Meier analysis demonstrated that the five-year overall survival rate of patients in the KIAA1199 negative or low expression group was considerably higher than that of the KIAA1199 high expression group (log-rank, $P<0.001$, Fig. 2).

Effect of KIAA1199 silencing on EOC cell migration and invasion

Western blot analysis was performed to detect KIAA1199 protein level among different EOC cell lines HO-8910, A2780, SK-OV3, and OVCAR-3 to investigate if the malignant phenotype of EOC was associated with abnormal KIAA1199 expression. Results showed that KIAA1199 protein level in cell lines HO8910 and CAOV-3 was lower than that in A2780 and OVCAR-3 (Figs. 3A and 3B).

Subsequently, we utilized the A2780 cell line to demonstrate cell biology experiments including cell migration and invasion. Four different shRNAs as well as a negative control were transfected into A2780 cells, while KIAA1199 expression was reduced drastically in shRNAs transfected A2780 cells compared with shRNA-NCtransfected cells. Matrigel-coated transwell assays indicated that KIAA1199 silencing degraded the migration and invasion ability of EOC cells (Fig. 3C).

Correlation between IL-6 stimulation and Cryptotanshinone disposal and KIAA1199 expression in EOC

To gain further insights into what could be the potential mechanism of KIAA1199 induced-advanced biological behavior on EOC cells, we performed predictions using ALGGEN-PROMO transcription factor binding software. It was predicted that there was potential STAT3 binding element on KIAA1199 promoter. Therefore, KIAA1199 could target components of IL-6/STAT3 pathway (Fig. 4A). To demonstrate the hypothesis, experiments were conducted by activating IL-6/STAT3 signal with IL-6 treatment and inhibiting with Cryptotanshinone. We chose HO8910 cells (KIAA1199 protein low expression) to perform the next work. First, four different cells groups (A, HO8910; 

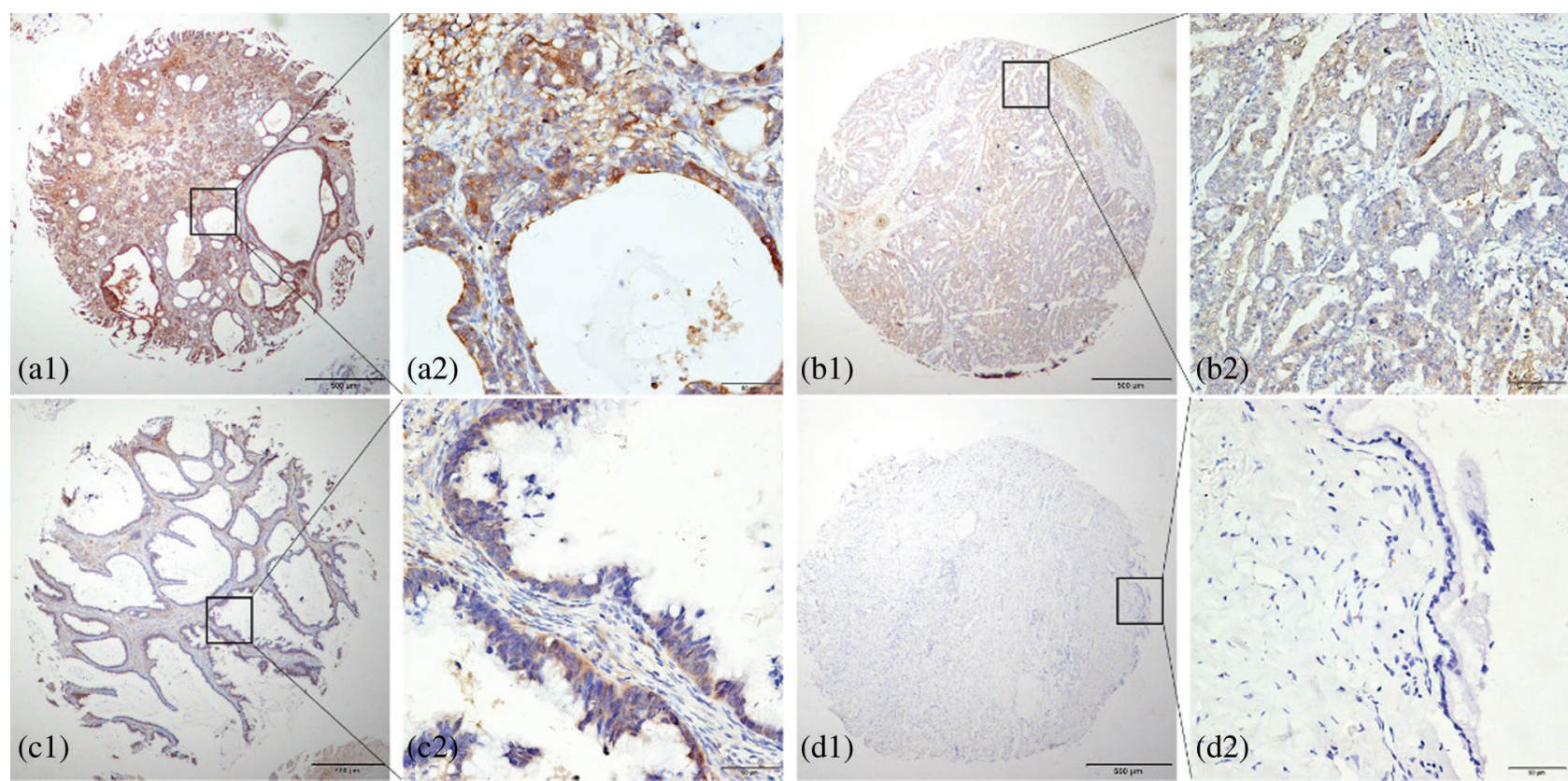

(b1)

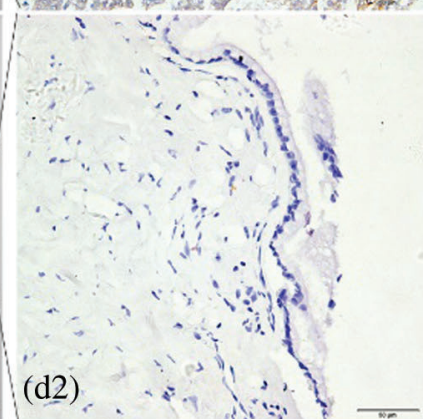

FIGURE 1. Immunohistochemical analysis of KIAA1199 protein expression in ovarian cancer (a, b), borderline ovarian epithelial tumor (c) and normal ovarian tissue $(\mathrm{d})$.

TABLE 1

Expression of KIAA1199 in EOC tissues and non-malignant tissues

\begin{tabular}{llllllll}
\hline & N & - & + & ++ & +++ & Pearson 2 & $P$ \\
\hline Normal ovarian tissue & 10 & 8 & 2 & 0 & 0 & 28.270 & $0.000^{*}$ \\
Borderline ovarian epithelial tumor & 36 & 14 & 9 & 8 & 5 & & \\
epithelial ovarian cancer & 126 & 20 & 33 & 35 & 38 & \\
\hline
\end{tabular}

Note: ${ }^{*} P<0.05$

TABLE 2

Correlation analysis of KIAA1199 expression and clinical pathological parameters of patients

\begin{tabular}{|c|c|c|c|c|c|c|c|}
\hline & $\mathbf{N}$ & - & + & ++ & +++ & Pearson 2 & $P$ \\
\hline \multicolumn{8}{|l|}{ Age (years) } \\
\hline$<52$ & 57 & 8 & 14 & 17 & 18 & 0.554 & 0.907 \\
\hline$\geq 52$ & 69 & 12 & 19 & 18 & 20 & & \\
\hline \multicolumn{8}{|l|}{ Tumor stage } \\
\hline FIGOI & 26 & 16 & 5 & 5 & 0 & 67.587 & $0.000^{*}$ \\
\hline FIGOII & 37 & 2 & 12 & 16 & 7 & & \\
\hline FIGOIII & 56 & 2 & 15 & 13 & 26 & & \\
\hline FIGOIV & 7 & 0 & 1 & 1 & 5 & & \\
\hline \multicolumn{8}{|l|}{ Histologic type } \\
\hline serous papillary cancer & 84 & 11 & 20 & 28 & 25 & 15.920 & 0.195 \\
\hline mucinous cancer & 18 & 1 & 6 & 3 & 8 & & \\
\hline endometrial cancer & 15 & 6 & 5 & 2 & 2 & & \\
\hline clear cell cancer & 5 & 1 & 2 & 1 & 1 & & \\
\hline transitional cell carcinoma & 4 & 1 & 0 & 1 & 2 & & \\
\hline
\end{tabular}


Table 2 (continued).

\begin{tabular}{|c|c|c|c|c|c|c|c|}
\hline & $\mathbf{N}$ & - & + & ++ & +++ & Pearson 2 & $P$ \\
\hline \multicolumn{8}{|c|}{ Tumor grade } \\
\hline G1 & 38 & 7 & 12 & 10 & 9 & 5.803 & 0.446 \\
\hline G2 & 31 & 5 & 11 & 6 & 9 & & \\
\hline G3 & 57 & 8 & 10 & 19 & 20 & & \\
\hline \multicolumn{8}{|c|}{ ascites } \\
\hline no & 29 & 8 & 4 & 9 & 8 & 5.693 & 0.128 \\
\hline yes & 97 & 12 & 29 & 26 & 30 & & \\
\hline \multicolumn{8}{|c|}{ Lymphatic invasion } \\
\hline no & 104 & 19 & 25 & 31 & 29 & 5.113 & 0.164 \\
\hline yes & 22 & 1 & 8 & 4 & 9 & & \\
\hline \multicolumn{8}{|c|}{ Peritoneal dissemination } \\
\hline no & 74 & 19 & 19 & 25 & 11 & 27.108 & $0.000^{*}$ \\
\hline yes & 52 & 1 & 14 & 10 & 27 & & \\
\hline \multicolumn{8}{|c|}{ Liver metastasis } \\
\hline no & 103 & 20 & 28 & 29 & 26 & 9.229 & $0.026^{*}$ \\
\hline yes & 23 & 0 & 5 & 6 & 12 & & \\
\hline
\end{tabular}

Note: ${ }^{\star} P<0.05$

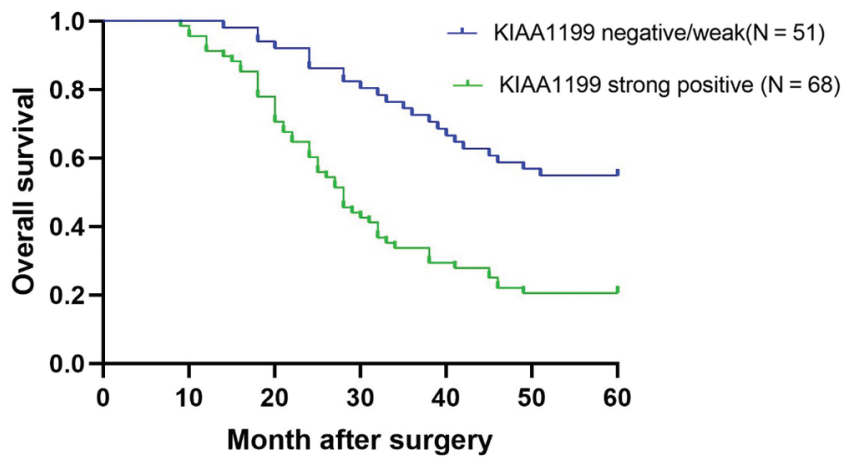

FIGURE 2. Kaplan-Meier plots showing relationship between KIAA1199 expression and clinical prognosis.

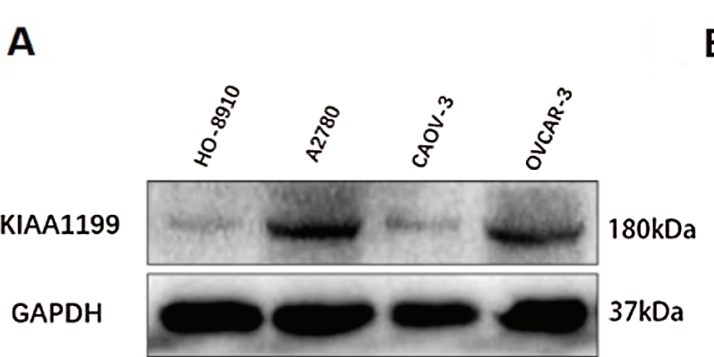

C

Ctrl

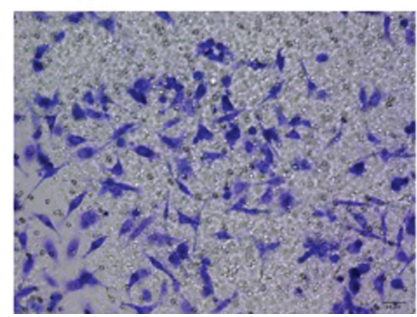

ShRNA-NC

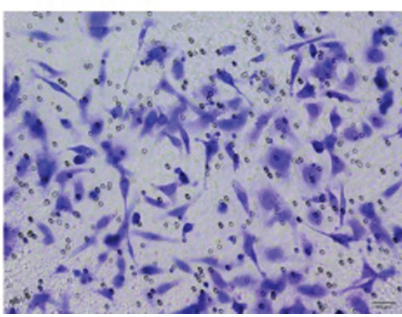

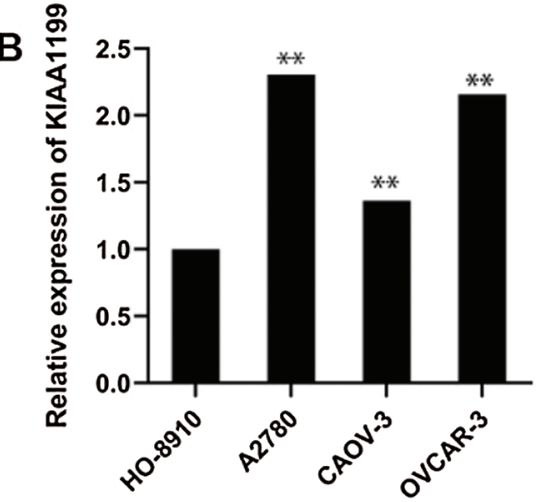

shRNA-KIAA1199

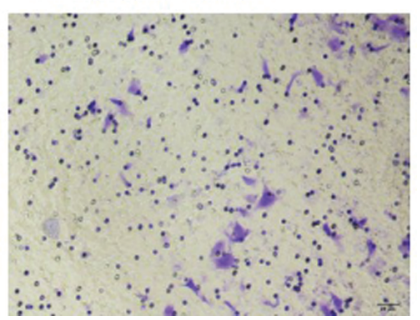

FIGURE 3. (A) Western blot analysis of the expression of KIAA1199 in HO-8910, A2780, CAOV-3, and OVCAR-3 cells. (B) Bar graph of the quantified data from A. (C) Images showing after $24 \mathrm{~h}$-shRNAKIAA1199 transfecting into A2780 cells. 
A

$-870$

$-840$

ACTTTTCCTACTGGTACTGTTTCCTCCCGCGAGGGAATGTCTAGA--/

$-740$

$-690$

/--CCACAGGGAATGTT TTTCTAACTT--//--TTAGAACTTGGACTTTCCTGATC--/

$-660$

$-620$

/--TTCTAGTGAGGAAGCATCACAGAACCCATTTTCCTGATGAGGAACTGAG-/

$-300$

$+120$

/-GGCGCGTGGAGGGAAGTTTC-//-ACCCAGATTTCCCAGACTAG

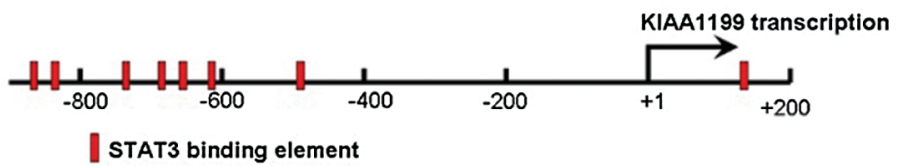

B

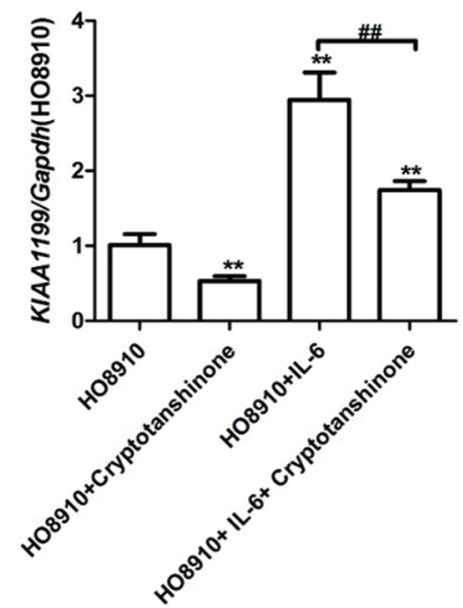

C
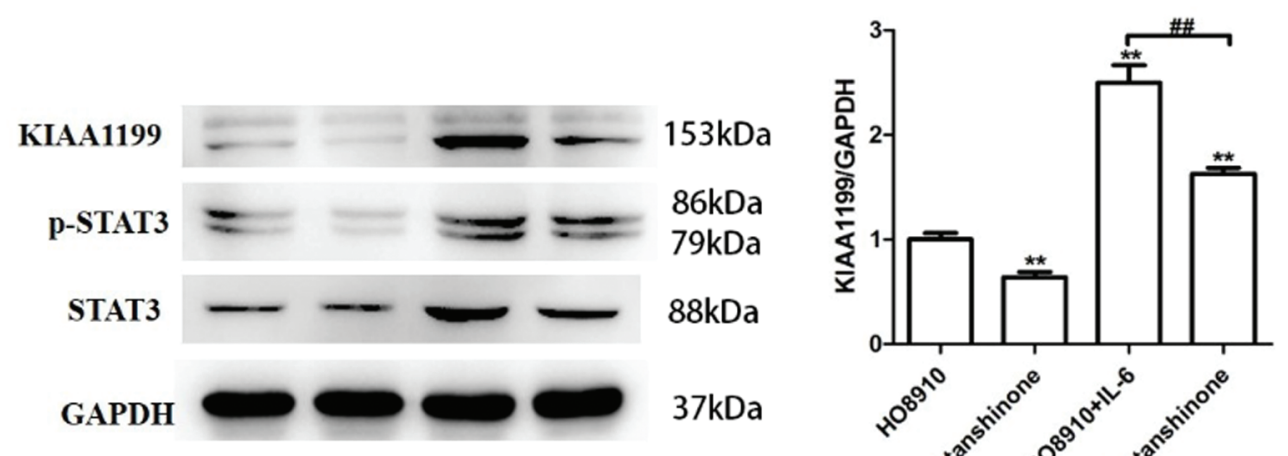

GAPDH
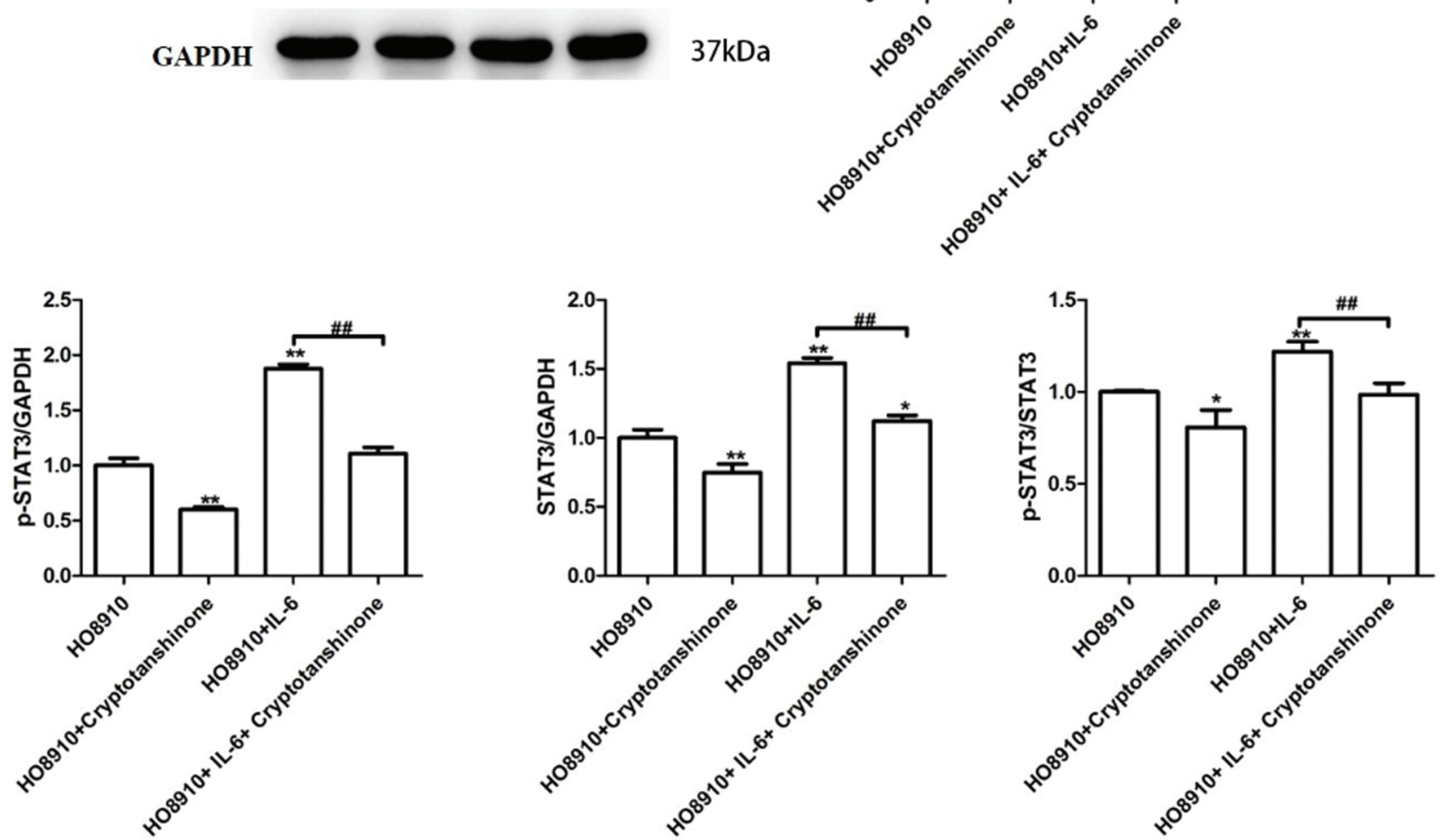

FIGURE 4. (A) KIAA1199 promoter potential target on IL-6/STAT3 pathway using ALGGEN-PROMO transcription factor binding software. (B) qRT-PCR result proved that reduced KIAA1199 expression after Cryptotanshinone disposal and increased KIAA1199 expression after IL-6 stimulation. Simultaneously with Cryptotanshinone, the suppressive effect on KIAA1199 expression was of significance, in comparison with the IL-6 stimulation group only $(P<0.01)$. (C) Western blot analysis showed that after Cryptotanshinone disposal or IL- 6 stimulation only, KIAA1199, p-STAT3, STAT3 expression were significantly reduced or increased separately $(P<0.01)$. Treatment of IL-6 simultaneously with Cryptotanshinone, KIAA1199 expression levels were significantly increased compared with HO-8910 group and reduced compared with HO-8910 + IL-6 group $(P<0.01)$.

B, HO8910+10 $\mu \mathrm{M}$ Cryptotanshinone (Zhu et al., 2020); C, HO8910+200 ng/mL IL-6; D, HO8910+200 ng/mL IL-6+10 $\mu \mathrm{M}$ Cryptotanshinone) were generated to compare
KIAA1199 expression level after IL-6 stimulation and Cryptotanshinone disposal. The results of qRT-PCR proved the expression of KIAA1199 was remarkably reduced after 
Cryptotanshinone disposal only in comparison with the HO8910 control group ( $P<0.01$, Fig. 4B). Contrariwise, the expression of KIAA1199 was significantly increased after IL-6 stimulation only. Simultaneously with Cryptotanshinone, the suppressive effect on KIAA1199 expression was of significance, in comparison with the IL-6 stimulation group only ( $P<0.01$, Fig. 4B). Similarly, the results of Western blot analysis showed that compared with the HO8910-NC, after Cryptotanshinone disposal or IL-6 stimulation only, the expression levels of KIAA1199, p-STAT3, STAT3 were significantly reduced or increased separately $(P<0.01$, Fig. $4 \mathrm{C}$ ). While treatment of Cryptotanshinone the same time with IL-6, KIAA1199 and STAT3 expression levels were significantly increased compared with group A and reduced compared with group $\mathrm{C}(P<0.01$, Fig. $4 \mathrm{C})$. But p-STAT3 expression and the value of p-STAT3/STAT3 were not of significantly different between group $\mathrm{D}$ and group $\mathrm{A}$.

KIAA1199 induces advanced biological behavior on EOC cells through activation of the IL-6/STAT3 pathway

To determine whether KIAA1199 induces advanced biological behavior on EOC cells through activation of the IL-6/STAT3 pathway, we used Chromatin immunoprecipitation (CHIP) assay to examine the mRNA expression of KIAA1199 after HO8910 cells DNA extracted from chromatin fragments immunoprecipitated with STAT3 antibody. Oligonucleotides used in this work were designed as follows (http://jaspar. genereg.net/search?advanced=true). KIAA1199-CHIP primer: forward 5'-TCTCCATTTGGGCCAAATGCCC-3'; reverse 5'GTACACACTAGCTCCGGGACT-3'. GAPDH-CHIP primer: forward 5'-TGATGCCCCCATGTTCGTCA-3'; reverse 5'AGGGGCCATCCACAGTCTTC-3’. Using DNA extracted from chromatin fragments immunoprecipitated with STAT3 antibody as templates, qRT-PCR amplified a 437bp fragment which containing the predicted binding site in the KIAA1199 promoter region, and the results showed that the target fragment was successfully amplified. KIAA1199-CHIP expression in group $\mathrm{C}$ was remarkably higher than that in group A (Fig. 5), indicating that STAT3 and KIAA1199 promoters combined together, and with the treatment of IL-6 the effect of combination was strengthened.

\section{Discussion}

EOC is the most lethal gynecological tumor (Gogineni et al., 2021). Since EOC has no specific clinical symptoms, it is mostly found in the late stage, which is one of the main factors contributing to its high mortality and morbidity. Due to the minimal effect of screening, it has been reported that the five-year relative survival rate for advanced patients was $29 \%$, and $92 \%$ in the early stage (Reid et al., 2017). Despite through the effort that the first-line treatment focuses on surgery, chemotherapy follow-up and systemic treatment such as molecularly targeted drugs and antibodies, 5 -year survival rate for EOC patients still remains beneath $20 \%$. Resistance is frequently an important reason, as many factors (Azais et al., 2020; Cong et al., 2020; Xu et al., 2020; Zhang et al., 2021) that make EOC are usually untreatable. Therefore, studying the mechanism that affects the occurrence and development of ovarian cancer is essential for finding novel and reliable treatments for ovarian cancer.

We detected that KIAA1199, an oncogenic protein, was highly expressed in ovarian malignant epithelial tumors, while EOC patients with high expression of KIAA1199 suffered a poor overall survival rate. Here, we attempted to elucidate the role of KIAA1199 in tumorigenesis of EOC in our present study. It is reported that KIAA1199, a transmembrane protein, expressed in various types of malignant tumor (Liu et al., 2018; Tang et al., 2018; Wang et al., 2020; Zhai et al., 2020; Zhao et al., 2019). Some research data presented that KIAA1199 gene was highly expressed in malignant tumors and could play a major role in cell mortality of normal human cells, thus combating cell immortality and tumorigenesis.

Studies have confirmed that the activation of IL-6/STAT3 pathway plays a vital role in the development of esophageal cancer (Sugimura et al., 2012), breast cancer (Ibrahim et al., 2017), colon cancer (Heichler et al., 2020), and gastric cancer (Ham et al., 2019). IL-6/STAT3 pathway is aberrantly hyperactivated and can boost to drive the proliferation, survival, invasion, and metastasis of tumor cells. However, the mechanism of KIAA1199 and IL-6/STAT3 pathway in ovarian cancer has not been confirmed. To better understand the relationship between KIAA1199 and IL-6/STAT3 pathway in EOC, we explored in this study that blockade of the IL-6/STAT3 signaling pathway and target molecules KIAA1199 in EOC might be a

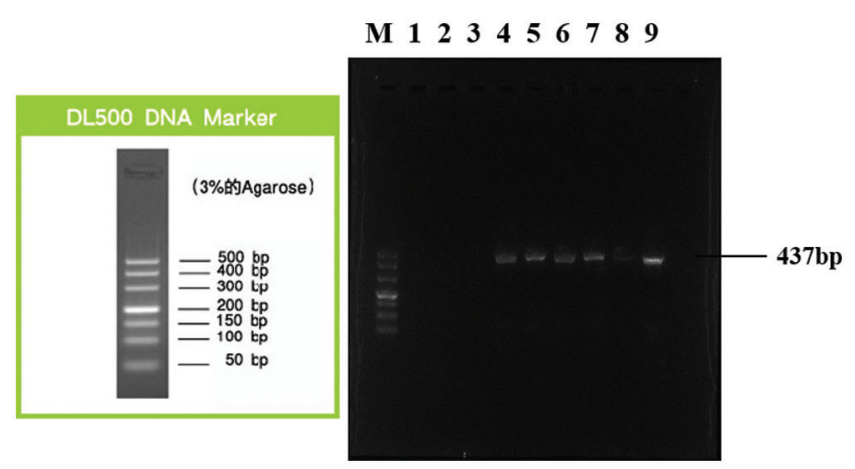

FIGURE 5. KIAA1199-CHIP expression in HO-8910 + IL-6 group was remarkably higher than that in HO-8910 group, indicating that STAT3 and KIAA1199 promoters combined together, and with the treatment of IL-6 the effect of combination was strengthened. (A) Lane M: DL500 DNA Marker; Lane 1: Negative control (sterile water); Lane 2: IgG immunoprecipitated DNA (HO-8910 group); Lane 3: IgG immunoprecipitated DNA (HO8910 + IL-6 group); Lane 4: DNA immunoprecipitated with acetylated histone $\mathrm{H} 3$ antibody (HO-8910 group); Lane 5: DNA immunoprecipitated with acetylated histone $\mathrm{H} 3$ antibody (HO8910 + IL-6 group); Lane 6: Input (HO-8910 group); Lane 7: Input (HO-8910 + IL-6 group). (B) Lane M: DL500 DNA Marker; Lane 1: Negative control (sterile water); Lane 2: IgG immunoprecipitated DNA (HO-8910 group); Lane 3: IgG immunoprecipitated DNA (HO-8910 + IL-6 group); Lane 4: DNA immunoprecipitated with acetylated histone $\mathrm{H} 3$ antibody (HO-8910 group); Lane 5: DNA immunoprecipitated with acetylated histone $\mathrm{H} 3$ antibody (HO8910 + IL-6 group); Lane 6: Input (HO-8910 group); Lane 7: Input (HO-8910 + IL-6 group); Lane 8: Chromatin DNA precipitated by STAT3 (HO-8910 group); Lane 9: Chromatin DNA precipitated by STAT3 (HO-8910 + IL-6 group). 
promising strategy to improve the prognosis for EOC patients. To verify the correlation, we examined the mRNA and protein expression of KIAA1199 after activating and inhibiting IL-6/STAT3 pathway by qRT-PCR and western blotting. IL-6 was used to activate IL-6/STAT3 pathway, and the expression levels of KIAA1199, STAT3 and p-STAT3 in EOC cells were increased after IL-6 stimulation. Furthermore, Cryptotanshinone disposal was utilized to inhibit IL-6/STAT3 pathway, which significantly reduced KIAA1199, p-STAT3, and STAT3 expression levels. Our data also showed that pathway inhibitor Cryptotanshinone treatment partially suppressed the STAT3 expression induced by IL-6. These results could show that KIAA1199 was transcriptionally activated by IL6/STAT3 pathway, thereby accelerating the deterioration of EOC. As we know, the IL-6/STAT3 pathway hyperactivation is generally associated with strong supression of the antitumor immune response (Kang et al., 2019). Therefore, treatments that target KIAA1199 may be used as an adverse prognosis factor and potential target, even to provide inhibiting tumor cell growth and metastasis and invasion by stimulating antitumor immunity.

In conclusion, our study proved that abnormal KIAA1199 expression may develop migration and invasive potential of EOC, be activated by IL6/STAT3 pathway, and boost tumor development, progression, and metastasis. Thus, KIAA1199 might act as a potential biomarker to identify a subgroup with a more aggressive phenotype and can be also used as a poor prognostic factor and potential therapeutic target for EOC. Nevertheless, there are also a few limitations of our study that we have to admit. For example, only vitro experiments have been involved. Hence, it is urgent for us to carry out further study and investigation, so as to confirm if KIAA1199 modulates EOC behavior through IL6/STAT3 pathway with EOC animal model and make further efforts to evaluate the clinical value of IL6/STAT3 pathway inhibitor in EOC.

Availability of Data and Materials: The datasets used and/or analyzed during the current study are available from the corresponding author on reasonable request.

Author Contribution: The authors confirm contribution to the paper as follows: study conception and design: Yunzhao $\mathrm{Xu}$, Songlin Zhou; data collection: Sainan Gao, Qi Meng, Yan Li; analysis and interpretation of results: Bing Lu, Zhen Wang; perform experiments and draft manuscript: Shuting $\mathrm{Gu}$, Jingyi Qin. All authors reviewed the results and approved the final version of the manuscript.

Ethics Approval: The experiment had received the approval from the ethics committee of the Affiliated Hospital of Nantong University (No. 2018-L005, DATE: 05/03/2018).

Funding Statement: The National Natural Science Foundation of China, Grant/Award No. 81802606.

Conflicts of Interest: The authors declare that they have no conflicts of interest to report regarding the present study.

\section{References}

Abe S, Usami S, Nakamura Y (2003). Mutations in the gene encoding KIAA1199 protein, an inner-ear protein expressed in Deiters' cells and the fibrocytes, as the cause of nonsyndromic hearing loss. Journal of Human Genetics 48: 564-570.

Arend RC, Jackson-Fisher A, Jacobs IA, Chou J, Monk BJ (2021). Ovarian cancer: new strategies and emerging targets for the treatment of patients with advanced disease. Cancer Biology \& Therapy 22: 89-105.

Azais H, Vignion-Dewalle AS, Carrier M, Augustin J, Da Maia E et al. (2020). Microscopic peritoneal residual disease after complete macroscopic cytoreductive surgery for advanced high grade serous ovarian cancer. Journal of Clinical Medicine 10: 41.

Cong S, Guo Q, Cheng Y, He Y, Zhao X et al. (2020). Immune characterization of ovarian cancer reveals new cell subtypes with different prognoses, immune risks, and molecular mechanisms. Frontiers in Cell and Developmental Biology 8: 614139 .

Da Silva AC, Jammal MP, Crispim PCA, Murta EFC, Nomelini RS (2020). The role of stroma in ovarian cancer. Immunological Investigations 49: 406-424.

Garbers C, Aparicio-Siegmund S, Rose-John S (2015). The IL-6/gp130/ STAT3 signaling axis: Recent advances towards specific inhibition. Current Opinion in Immunology 34: 75-82.

Gogineni V, Morand S, Staats H, Royfman R, Devanaboyina M et al. (2021). Current ovarian cancer maintenance strategies and promising new developments. Journal of Cancer 12: 38-53.

Guan LY, Lu Y (2018). New developments in molecular targeted therapy of ovarian cancer. Discovery Medicine 26: 219-229.

Ham IH, Oh HJ, Jin H, Bae CA, Jeon SM et al. (2019). Targeting interleukin-6 as a strategy to overcome stroma-induced resistance to chemotherapy in gastric cancer. Molecular Cancer 18: 68.

Heichler C, Scheibe K, Schmied A, Geppert CI, Schmid B et al. (2020). STAT3 activation through IL-6/IL-11 in cancer-associated fibroblasts promotes colorectal tumour development and correlates with poor prognosis. Gut 69: 1269-1282.

Ibrahim SA, Gadalla R, El-Ghonaimy EA, Samir O, Mohamed HT et al. (2017). Syndecan-1 is a novel molecular marker for triple negative inflammatory breast cancer and modulates the cancer stem cell phenotype via the IL-6/STAT3, Notch and EGFR signaling pathways. Molecular Cancer 16: 57.

Johnson DE, O'keefe RA, Grandis JR (2018). Targeting the IL-6/JAK/ STAT3 signalling axis in cancer. Nature Reviews Clinical Oncology 15: 234-248.

Kang S, Tanaka T, Narazaki M, Kishimoto T (2019). Targeting interleukin-6 signaling in clinic. Immunity 50: 1007-1023.

Kitamura H, Ohno Y, Toyoshima Y, Ohtake J, Homma S et al. (2017). Interleukin-6/STAT3 signaling as a promising target to improve the efficacy of cancer immunotherapy. Cancer Science 108: 1947-1952.

Kroeger PT, Jr., Drapkin R (2017). Pathogenesis and heterogeneity of ovarian cancer. Current Opinion in Obstetrics \& Gynecology 29: 26-34.

Lee JM, Minasian L, Kohn EC (2019). New strategies in ovarian cancer treatment. Cancer 125: 4623-4629.

Lheureux S, Gourley C, Vergote I, Oza AM (2019). Epithelial ovarian cancer. Lancet 393: 1240-1253.

Li L, Yan LH, Manoj S, Li Y, Lu L (2017). Central role of CEMIP in tumorigenesis and its potential as therapeutic target. Journal of Cancer 8: 2238-2246.

Liu J, Han P, Gong J, Wang Y, Chen B et al. (2018). Knockdown of KIAA1199 attenuates growth and metastasis of hepatocellular carcinoma. Cell Death Discovery 4: 102. 
Mihara M, Hashizume M, Yoshida H, Suzuki M, Shiina M (2012). IL-6/IL-6 receptor system and its role in physiological and pathological conditions. Clinical Science 122: 143-159.

Nimmagadda S, Penet MF (2019). Ovarian cancer targeted theranostics. Frontiers in Oncology 9: 1537.

Reid BM, Permuth JB, Sellers TA (2017). Epidemiology of ovarian cancer: A review. Cancer Biology \& Medicine 14: 9-32.

Rooth C (2013). Ovarian cancer: Risk factors, treatment and management. British Journal of Nursing 22: S23-S30.

Sheng $\mathrm{N}, \mathrm{Xu} \mathrm{YZ}, \mathrm{Xi} \mathrm{QH}$, Jiang HY, Wang CY et al. (2018). Overexpression of KIF2A is suppressed by miR-206 and associated with poor prognosis in ovarian cancer. Cellular Physiology \& Biochemistry 50: 810-822.

Soroosh A, Albeiroti S, West GA, Willard B, Fiocchi C, De La Motte CA (2016). Crohn's disease fibroblasts overproduce the novel protein KIAA1199 to create proinflammatory hyaluronan fragments. Cellular and Molecular Gastroenterology and Hepatology 2: 358-368.

Sugimura K, Miyata H, Tanaka K, Hamano R, Takahashi T et al. (2012). Let-7 expression is a significant determinant of response to chemotherapy through the regulation of IL-6/STAT3 pathway in esophageal squamous cell carcinoma. Clinical Cancer Research 18: 5144-5153.

Tang Z, Ding Y, Shen Q, Zhang C, Li J et al. (2018). KIAA1199 promotes invasion and migration in non-small-cell lung cancer (NSCLC) via PI3K-Akt mediated EMT. Journal of Molecular Medicine 97: 127-140.

Wang A, Zhu J, Li J, Du W, Zhang Y et al. (2020). Downregulation of KIAA1199 by miR-486-5p suppresses tumorigenesis in lung cancer. Cancer Medicine 9: 5570-5586.

Xu S, Yu C, Ma X, Li Y, Shen Y et al. (2020). IL-6 promotes nuclear translocation of HIF-1alpha to aggravate chemoresistance of ovarian cancer cells. European Journal of Pharmacology 894: 173817.

Yang Y, Yang Y, Yang J, Zhao X, Wei X (2020). Tumor microenvironment in ovarian cancer: Function and therapeutic strategy. Frontiers in Cell and Developmental Biology 8: 758.

Yoshida H, Nagaoka A, Nakamura S, Tobiishi M, Sugiyama Y, Inoue $S$ (2014). N-Terminal signal sequence is required for cellular trafficking and hyaluronan-depolymerization of KIAA1199. FEBS Letters 588: 111-116.

Yoshida H, Okada Y (2019). Role of HYBID (Hyaluronan binding protein involved in hyaluronan depolymerization), alias KIAA1199/CEMIP, in hyaluronan degradation in normal and photoaged skin. International Journal of Molecular Sciences 20: 5804.

Zhai XW, Ma Y, Zeng Y, Dou D, Fan H et al. (2020). Serum KIAA1199 is an advanced-stage rognostic biomarker and metastatic ncogene in cholangiocarcinoma. Sedentary Life and Nutrition 12: 23761.

Zhang Y, Jia S, Jiang WG (2014). KIAA1199 and its biological role in human cancer and cancer cells. Oncology Reports 31: 1503-1508.

Zhang Y, Shi X, Zhang J, Chen X, Zhang P et al. (2021). A comprehensive analysis of somatic alterations in Chinese ovarian cancer patients. Scientific Reports 11: 387.

Zhao L, Zhang D, Shen Q, Jin M, Lin Z et al. (2019). KIAA1199 promotes metastasis of colorectal cancer cells via microtubule destabilization regulated by a PP2A/stathmin pathway. Oncogene 38: 935-949.

Zhu W, Liu C, Lu T, Zhang Y, Zhang S et al. (2020). Knockout of EGFL6 by CRISPR/Cas9 mediated inhibition of tumor angiogenesis in ovarian cancer. Frontiers in Oncology 10: 1451. 